\title{
Progression to vascular dementia of patients with mild cognitive impairment: relevance of mild parkinsonian signs
}

\author{
Marco Mauri \\ Simona Corbetta \\ Cristina Pianezzola \\ Elena Ambrosoni \\ Giulio Riboldazzi \\ Giorgio Bono \\ Unit of Neurology, Dept \\ of Clinical Medicine, Ospedale di \\ Circolo - University of Insubria, \\ Varese, Italy
}

Correspondence: Marco Mauri UO Neurologia - Ospedale di Circolo, Università dell'Insubria, Viale Borri 57, 21100 Varese, Italy

Tel +390332278427

Fax +390332 393184

Email marco.mauri@uninsubria.it

\begin{abstract}
Mild parkinsonian signs (MPS) may be found among patients presenting with mild cognitive impairment (MCI), but few data are available about the relation of these signs with the prospective risk for dementia. Our retrospective investigation considered a case-series of 119 MCI subjects followed over a three-year period: their baseline clinical picture has been analyzed in search of correlation between the cognito-motor profile and the final diagnosis. The population included 66 patients with amnesic MCI and 53 with an involvement of other cognitive areas (nonamnesic MCI). MPS were detected in 22 subjects (18.5\%). At the first observation, MPS cases showed an higher frequency of nonamnesic MCI and more pronounced deficits at the Trail Making Test $(\mathrm{p}<0.05)$. After a three-year follow-up, 48 patients had converted to dementia. The presence of MPS at the baseline evaluation was significantly related to the development of a vascular-type dementia. The study investigates the association between MPS and MCI and might indicate for these cases a greater risk for an involvement of executive functions and the subsequent development of vascular dementia.
\end{abstract}

Keywords: mild cognitive impairment, dementia, cerebrovascular disease, parkinsonism

\section{Introduction}

Parkinsonian signs such as gait disturbances, rigidity, bradykinesia and tremor are common among older adults and significantly associated with negative outcomes such as dementia and death (Wilson et al 2002; Louis et al 2004). A recent study shows that mild parkinsonian signs (MPS), especially rigidity, may frequently be observed among patients with amnestic mild cognitive impairment (MCI) and that these two conditions may share a common pathogenesis (Louis et al 2005). In an another study, subjects with MCI showed associated parkinsonian signs more frequently than controls without cognitive impairment. Among patients with MCI, lower levels of cognitive functioning, particularly in perceptual speed, were also associated with higher levels of parkinsonism (Boyle et al 2005). In contrast to previous finding of a unique association between amnestic MCI and parkinsonism, the study by Boyle and colleagues (2005) suggests that parkinsonian signs may be more prominent among individuals with nonamnestic forms of MCI (ie, with involvement of multiple areas). Moreover the association between MCI and parkinsonism is not completely explained by vascular risk factors or vascular disease.

A longitudinal cohort study involving older Catholic clergy membership concluded that persons with MCI also have impaired motor function, and the degree of impairment in lower extremity function is related to risk of Alzheimer's disease (Aggarwal et al 2006).

The basis for these signs is unclear, but is likely to be multifactorial, including an age-associated decline in dopaminergic nigrostriatal activity, the early development of neurodegenetative (Lewy body or Alzheimer's diseases) pathologies in the basal 
ganglia, or the accumulations of vascular pathology in the brain (Louis and Bennet 2007). Recent neuropathological studies indicate that neuronal loss in substantia nigra, partly related to tau lesions, is a major pathological substrate of extrapyramidal signs (EPS) in Alzheimer's disease (AD). EPS in elderly patients may be a surrogate marker for severe neuritic AD pathology (Attems et al 2007).

On the other hand, impairment of cognitive function has been demonstrated even in newly diagnosed Parkinson disease patients (PD), with deficits being most prominent in the domains of memory and executive functions; also, an older age at disease onset is likely to be an important determinant of cognitive dysfunction in PD (Muslimovic et al 2005).

Based on these reports, we have tried to investigate how the association between MCI and MPS may be related to the evolution towards different type of dementias or other neurodegenerative disorders.

\section{Methods}

In this retrospective investigation we considered a caseseries of subjects fitting the criteria for MCI (Petersen et al 2001), folllowed over a three-year period. During this time, a population of 135 consecutive cases was observed but, at the present moment, 16 subjects were lost at follow-up (7 died and 9 were not evaluated for poor compliance). The analysis was conducted on 119 cases (73.1 \pm 5.7 years, M/F 50/69). The participants involved in this study were recruited from outpatients attending the Laboratory of Neuropsychology and the Alzheimer's Unit (UVA) at the Ospedale di Circolo and Fondazione Macchi in Varese, Italy.

After the first examination, MCI subjects were evaluated every 12 months to detect the patients who converted to dementia. We considered the baseline clinical picture in search of correlation between cognito-motor profile and the final diagnosis of dementia, as defined by the current clinical criteria (McKhan et al 1984; Morris 1993; The Lund and Manchester Groups 1994; Erkinjuntti et al 2000; McKeith et al 2005).

All cases underwent, at the baseline examination, a standard neurological examination, laboratory tests (routine blood samples, thyroid, B12 and folate, Venereal Disease Research Laboratory), neuroimaging investigation (brain computed tomography [CT]) in order to exclude secondary causes of brain organic dysfunction. The use of psychiatric drugs that may affect cognition or motor functions (such as antipsychotics or anticholinergic) was also excluded.

A validated CT-based weighted rating scale sensitive to detect different degrees of subcortical ischemic vascular disease (SIVD) was also calculated. This rating scale is valid and is sensitive to capture different degrees of SIVD associated with mild cognitive deterioration (Geroldi et al 2003).

Neurological examination included the motor portion of the Unified Parkinson Disease Rating Scale (UPDRS). MPS was determined by the presence of changes in axial function, rigidity or tremor (Louis et al 2004).

All the patients underwent a standardized neuropsychological battery, designed to assess cognitive functions that are generally involved in dementia: orientation, memory, attention, information processing speed, abstract reasoning, language and visuospatial ability. Tests administered included: Clinical Dementia Rating scale (CDR) (Morris 1993) for staging of dementia, Mini Mental State Examination (MMSE) (Folstein et al 1975), Trail Making Test part A and B (TMT) (Giovagnoli et al 1996), and Mental Deterioration Battery (MDB) (Carlesimo et al 1996). The MDB is comprised of eight tests, four are expressions from the processing of verbal material and four derive from the processing of visuospatial material. The verbal tests are: immediate (IR) and delayed recall (DR) of Rey's 15 words, word fluency (FAS), and phrase construction. The visuospatial tests are: Raven's 47 progressive colored matrices, immediate visual memory (IVM), and copying of drawings freehand (CD FH) and with landmarks (CD WL).

Affective symptoms were assessed using the Geriatric Depression Scale (GDS) (Yesavage et al 1983). GDS scores were used to exclude patients with severe depression and as control variable between the different groups. Instrumental activities of daily living (IADL) were also investigated (Lawton and Brody 1969).

\section{Statistical analysis}

Standardized and validated Italian language versions of all the tests are available. The cognitive performances of the patients were evaluated against normative data for the Italian population. Raw scores from each test were corrected for age, sex, and education and transformed into corrected scores, in accordance with Italian standardization studies.

A logistic regression analyses were performed using MPS status and type of dementia as the dependent variables and all the other relevant variables as predictors (SPSS version 13.0, SPSS Inc., Chicago, IL, USA).

\section{Results}

\section{Baseline evaluation}

Our population (119 cases of MCI) included, in according with the results of the baseline neuropsychological battery, $66(55.5 \%)$ subjects with selective memory impairment (amnesic MCI) and 53 (45.5\%) with an involvement of other 
cognitive areas (nonamnesic MCI). Among nonamnesic MCI the cognitive profile was characterized by a selective impairment of executive function or by a diffuse borderline performance.

According to Petersen criteria, MMSE scores, corrected by age and education, were within the normal range. At the time of the first examination study participants obtained a score equal to 0.5 (questionable dementia) at the CDR scale for staging of dementia, without significant impairment in social functioning (IADL).

MPS were detected in 22 subjects (18.5\%) with postural instability and gait difficulty in 16 patients and dominant tremor in 6 cases. The presence of MPS were more frequent $(\mathrm{p}<0.05)$ among nonamnesic MCI $(13 / 53)$ in respect to amnesic MCI (9/66). Demographic variables and cognitive performances among patients with (MPS+) and without MPS (MPS-) are reported in Table 1. At the baseline observation the analyses of cognitive profile showed a worse performance of the TMT (B-A) in the MPS+ group ( $<<0.05)$, without significant differences for the other cognitive, affective and functional variables.

No significant difference was detected for GDS score between the two groups.

Furthermore in these cases the evaluation of basal brain CT scan pointed out the presence of a greater presence of moderate to severe subcortical vascular abnormalities - SIVD (Figure 1) that, however, did not reached the statistical significance. The analysis of comorbility revealed an higher

Table I Demographic and clinical variables of patients with (MPS+) and without MPS (MPS-)

\begin{tabular}{lll}
\hline Demographic variables & MPS+ (N, 22) & MPS- (N, 97) \\
\hline Age & $74 \pm 6.1$ & $72.8 \pm 5.3$ \\
Sex m/f & $12 / 10$ & $41 / 56$ \\
Education & $6.9 \pm 3.1$ & $7.1 \pm 3.4$ \\
Dementia variables & & \\
GDS & $10.2 \pm 5.1$ & $8.7 \pm 4.4$ \\
IADL & $7.4 \pm 0.6$ & $7.6 \pm 0.4$ \\
MMSE & $26.9 \pm 1.6$ & $27.2 \pm 1.8$ \\
Comorbility & & \\
Hypertension & $11(50)$ & $31(32)$ \\
Diabetes & $2(9)$ & $5(5)$ \\
TIA/minor stroke & & $3(3)$ \\
Ischemic cardiopathy & $4(18)$ & $14(14.5)$ \\
\hline
\end{tabular}

Notes: ${ }^{*} \mathrm{p}<0.05$ Chi-square test; ${ }^{\circ}$ Events occurred before the onset of cognitive complaints thus excluding a direct cause-effect relationship.

Abbreviations: GDS, geriatric depression scale; IADL, instrumental activity of daily living; MMSE, mini mental state examination.

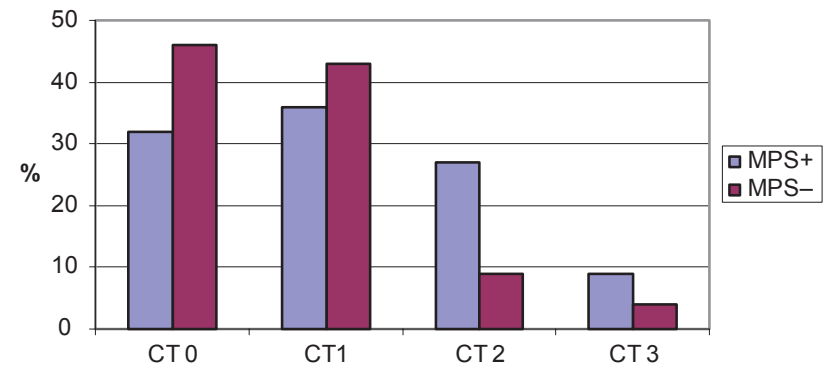

Figure I Distribution of computed tomography (CT)-based weighted rating scale for subcortical ischemic vascular disease (percentage of cases) among subjects with and without MPS.

Baseline CT score for SIVD: $O$ absent; I mild, 2 moderate, 3 severe degree of subcortical ischemic vascular disease.

presence of vascular risk factors for nonamnesic group with a significant difference $(\mathrm{p}<0.05)$ for previous TIA or lacunar syndromes. These events had occurred before the onset of cognitive complaints thus excluding a direct cause-effect relationship.

\section{Follow-up}

After a three-year follow-up 48/119 MCI patients (40.5\%) had converted to possible or probable dementia of different types: Alzheimer's disease (AD), 31 (64.5\%); Lewy Body dementia (LBD), 7 (14.5\%); vascular dementia, 8 (16.5\%); or fronto-temporal dementia (FTD), 2 (4.5\%). Two cases of MPS group did not develop a progression towards dementia but were diagnosed as idiopathic PD. The classification of different type of dementia was made on the basis of the criteria exposed in the material and methods. Brain CT or magnetic resonance imaging (MRI) scans were repeated when necessary to confirm the clinical features of vascular dementia.

The annual rate of conversion to dementia of the entire population is reported in Figure 2: the group with MPS showed a more evident trend to the development of dementia.

MPS was present, at the baseline evaluation in 4/31 cases of $\mathrm{AD}, 2 / 7$ of LBD, and 5/8 of vascular dementia. On this basis, the presence of MPS was significantly related to

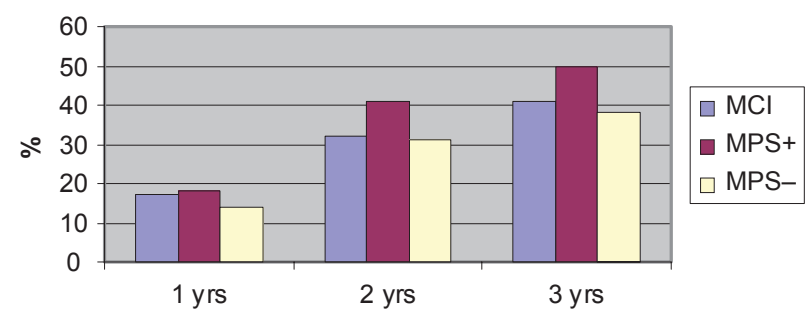

Figure 2 Cumulative rate of conversion to dementia over a three-year follow-up. Notes: $\mathrm{MCl}$ : all cases observed ( $\mathrm{N}, \mathrm{I}$ 19), MPS+ patient with mild parkinsonian signs (N, 22), MPS- patients without mild parkinsonian signs ( $N, 97)$. 
the development of vascular dementia $(p<0.05)$. On the other hand patients with vascular dementia showed, at the baseline brain CT scan, moderate to severe SIVD, whereas SIVD alterations were similar among AD and LBD groups. Neuropsychological performance of the vascular dementia group was characterized by worse scores at TMT at the baseline examination, which did not reached a significant value probably due to the very low number of cases.

No significant difference in the occurrence of MPS was detectable at the first evaluation between patients converted to $\mathrm{AD}$ and $\mathrm{LDB}$, the cognitive profile revealed only a worse performance in the copy of drawings among the LDB group.

\section{Discussion}

Mild cognitive impairment represents a condition characterized by different neuropsychological profile (amnesic, nonamnesic, multiple areas defects). During the last years several clinical and noncognitive aspects were also investigated such as comorbidity, the prevalence of psychiatric syndromes and symptoms (Apostolova and Cummings 2008) and, recently, the medical decision making-capacity of these subject (Okonkwo et al 2007). The present study focused the attention on the presence of MPS in a group of amnesic and nonamnesic MCI followed for a three-year period. Our data confirm the relevance of the association between MPS and MCI and might indicate an higher chance for these cases to develop dementing conditions related to vascular disease.

In the study by Louis and colleagues (2004), MPS, especially rigidity, are associated with amnestic MCI. Moreover the authors suggested that MPS and MCI may share similar pathogenesis. The observation that MPS were associated with vascular risk factors and vascular disease suggests that cerebrovascular disease may contribute to both conditions.

In contrast to this finding of a unique association between amnestic MCI and MPS, the results of Boyle and colleagues (2005) suggested that MPS may be more prominent among individuals with non-amnestic MCI as opposed to amnestic MCI. Another study demonstrated that the severity of MPS was related to the severity of cognitive impairment, in particular nonmemory functions, and also to a different pattern of psycho-behavioral symptoms (Rozzini et al 2007).

In our study, MPS occurred in 22 out of 119 cases $(18.5 \%)$ that differed from the rest of population for a greater impairment of executive function and, subsequently, a greater number of cases with nonamnesic MCI. In particular a pathological or borderline score at the TMT significantly divided the two groups with and without MPS. This association, as observed by Boyle and colleagues, could indicate that the involvement of executive functions is consistent with the dysfunction of fronto-subcortical systems and the development of parkinsonian signs (Wilson et al 2002).

Among MPS patients the analysis of movement disorders showed a prevalent presence of postural instability and gait disturbances (PIGD). With regard to this finding a recent study confirmed that PIGD motor subtype was associated with a faster rate of cognitive decline in PD and may be considered a risk factor for incident dementia in PD (Burn et al 2006).

Over a three-year period, $48 \mathrm{MCI}$ cases converted to dementia and the presence of MPS signs, at the baseline evaluation, is significantly related to the development of SIVD. Among these two groups, the presence of vascular abnormalities was, as expected, showed more evidence for vascular dementia. No difference between $\mathrm{AD}$ and LBD was detected.

A recent study on a large group of MCI patients confirmed that, in the context of pervasive episodic memory impairment, tests assessing the free recall of verbal material following a delay interval demonstrated the greater sensitivity to memory deficits of amnesic MCI subjects who developed AD (Perri et al 2007). On the contrary, strategic cerebrovascular lesions detected by MRI in patients with CDR score equivalent to 0.5 were associated with impaired TMT B performances. In particular executive dysfunction was probably based on an impaired fronto-subcortical circuit (Ishii et al 2007). In some vascular dementia subtypes, particularly those caused by subcortical microvascular disease, dementia may be preceded by MCI, which has similar domains of cognitive impairment and similar progressive course that may mimic AD (Meyer et al 1981).

Although a MRI examination was available only for few cases and a CT weighted scale was used, these findings also indicated that MCI with MPS is partly explained by the occurrence of vascular ischemic abnormalities.

Moreover during the follow-up period we observed several cases converted to different types of dementia including LBD, but 2 patients also developed a PD associated with MCI. Two different studies showed that, in newly diagnosed $\mathrm{PD}$, a cognitive dysfunction ranging from $24 \%$ to $36 \%$ was detectable (Foltynie et al 2004; Louis et al 2005). This finding could indicate that cognitive dysfunctions in early PD may reflects neuropathological changes that are distinct from those associated with motor disorders. 
The overlap between these different conditions suggests that the mechanisms underlying MPS are probably heterogeneous with a range of potential pathophysiological processes that include nerodegenerative and vascular pathologies (Louis and Bennet 2007).

\section{Disclosure}

The authors report no conflicts of interest in this work.

\section{References}

Aggarwal NT, Wilson RS, Beck TL, et al. 2006. Motor dysfunction in mild cognitive impairment and the risk of incident Alzheimer disease. Arch Neurol, 63:1763-9.

Apostolova LG and Cummings JL. 2008. Neuropsychiatric manifestation in mild cognitve impairment: a systematic review of the literature. Dement Geriatr Cogn Disord, 25:115-26.

Attems J, Quass M, Jellinger KA, et al. 2007. Tau and alpha-synuclein pathology in Alzheimer disease: relation with extrapyramidal signs. Acta Neuropathol, 113:53-62.

Boyle PA, Wilson RS, Aggarwal NT, et al. 2005. Parkinsonian signs in subjects with mild cognitive impairment. Neurology, 65:1901-6.

Burn DJ, Rowan EN, Allan LM, et al. 2006. Motor subtype and cognitive decline in Parkinson's disease, Parkinson' disease with dementia, and dementia with Lewy bodies. J Neurol Neurosurg Psychiatry, 77:585-9.

Carlesimo GA, Caltagirone C, Gainotti G, et al. 1996. The Mental Deterioration Battery: normative data, diagnostic reliability and qualitative analyses of cognitive impairment. Eur Neurol, 36:378-84.

Erkinjuntti T, Inzitari D, Pantoni L, et al. 2000. Research criteria for subcortical vascular dementia in clinical trials. J. Neural Transmit Suppl, 59:23-30.

Folstein MF, Folstein SE, McHugh PR, et al. 1975. Mini Mental State. A practical method for grading the cognitive state of patients for the clinician. J Psychiat Res, 12:189-98.

Foltynie T, Brayne CEG, Robbins TW, et al. 2004. The cognitive ability of an incident cohort of Parkinson's patients in the UK. The CamPaIGN Study. Brain, 127:550-60.

Geroldi C, Galluzzi S, Testa C, et al. 2003. Validation study of a CTbased weighted rating scale for subcortical ischemic vascular disease in patients with mild cognitive deterioration. Eur Neurol, 49:193-209.

Giovagnoli AR, Del Pesce M, Mascheroni S, et al. 1996. Trail making test: normative values from 287 normal adult controls. Ital J Neurol Sci, 17:305-9.
Ishii H, Meguro K, Yamaguchi S, et al. 2007. Prevalence and cognitive performances of vascular cognitive impairment no dementia in Japan: the Osaki-Tajiri Project. Eur J Neurol, 14:609-16.

Lawton MP and Brody EM. 1969. Assessment of older people selfmaintening and instrumental activities of daily living. Gerontologist, 9:179-86.

Louis ED and Bennet DA. 2007. Mild parkinsonian signs: an overview of an emerging concept. Mov Disord, 22:1681-8.

Louis ED, Schupf N, Manly J, et al. 2005. Association between mild parkinsonian signs and mild cognitive impairment in a community. Neurology, 64:1157-61.

Louis ED, Tang X, Mayeux R, et al. 2004. Parkinsonian signs in older people in a community-based study: risk of incident dementia. Arch Neurol, 61:1273-6.

McKeith I, Dickson DW, Lowe J, et al. 2005. Diagnosis and management of dementia with Lewy Body: third report of DLB Consortium. Neurology, 65:1863-72.

McKhan G, Drachman D, Folstein M, et al. 1984. Report of NINCDSADRDA Work Group under the auspices of Department of Health and Human Services. Neurology, 34:939-44.

Meyer JS, Xu G, Thornby J, et al. 2002. Is mild cognitve impairment prodromal for vascular dementia like Alzheimer's disease? Stroke, 33:1981-5.

Morris JC. 1993. The Clinical Dementia Rating (CDR): current version and scoring rules. Neurology, 43:2412-14.

Muslimovic D, Post B, Speelman JD, et al. 2005. Cognitive profile of patients with newly diagnosed Parkinson disease. Neurology, 65:1239-45.

Okonkwo O, Griffith HR, Belue K, et al. 2007. Medical decision-making capacity in patients with mild cognitive impairment. Neurology, 69:1528-35.

Perri R, Serra L, Carlesimo GA, et al. 2007. Amnestic mild cognitve impairment: difference of memory profile in subjects who converted or did not convert to Alzheimer's disease. Neuropsychology, 5:549-58.

Petersen R, Doody R, Kurz A, et al. 2001. Current concepts in mild cognitive impairment: clinical characterization and outcome. Arch Neurol, 58:1985-92.

Rozzini L, Chilovi BV, Bertoletti E, et al. 2007. Mild parkinsonian signs and psycho-behavioral symptoms in subjects with mild cognitive impairment. Int Psychogeriatr, 17:1-10.

The Lund and Manchester Groups. 1994. Clinical and neuropathological criteria for frontotemporal dementia. J Neurol Neurosurg Psychiatry, 57:416-8.

Wilson RS, Schneider JA, Beckett LA, et al. 2002. Progression of gait disorders and rigidity and risk of death in older persons. Neurology, 58:1815-19.

Yesavage JA, Brink TL, Rose TL, et al. Development and validation of a geriatric depression screening scale: a preliminary report. J Psychiatr Res, 17:37-49. 
